\title{
A prática esportiva e o assoalho pélvico feminino: uma revisão da literatura
}

\author{
The sports practice and the female pelvic floor: a literature review
}

\author{
Rafaela de Melo Silva ${ }^{1, *}$ \\ Maisa Paula dos Santos ${ }^{1}$ \\ Maita Poli Araújo² \\ Marair Gracio Ferreira Sartori ${ }^{2}$ \\ Ana Paula Magalhães Resende ${ }^{3}$
}

\section{Resumo}

Objetivos: a) Identificar a prevalência da incontinência urinária em atletas e praticantes de atividade física; b) Verificar o impacto da atividade física nos músculos do assoalho pélvico de atletas; e c) Averiguar as evidências científicas sobre o tratamento fisioterapêutico da incontinência urinária em atletas e praticantes de atividade física. Métodos: Foi realizada uma revisão crítica da literatura com buscas nas bases de dados Pubmed e Lilacs, nos últimos dez anos. Foram incluídos estudos experimentais que avaliaram a prevalência de incontinência urinária durante a prática esportiva, estudos sobre o impacto da atividade física nos músculos do assoalho pélvico e estudos de intervenção nas disfunções dos músculos do assoalho pélvico em atletas. Resultados: Foram incluídos 17 estudos. A prevalência da incontinência urinária variou entre $5 \%$ e $88,9 \%$, a depender do tipo de atividade física. A idade média das mulheres foi de 24,9 anos sendo a maioria nuligesta. No que se refere ao impacto da atividade física nos músculos do assoalho pélvico, os principais métodos de avaliação muscular utilizados foram a palpação vaginal, eletromiografia e ultrassonografia tridimensional. Por fim, em relação ao tratamento, foram encontrados três estudos de intervenção, tipo antes e depois, onde diferentes tipos de protocolos foram utilizados com objetivo de reabilitação dos músculos do assoalho pélvico em mulheres atletas com queixa de incontinência urinária, mostrando efetividade. Conclusões: A reabilitação muscular pareceu ser eficaz para a melhora da incontinência urinária, são necessários mais estudos randomizados para melhor comprovação da sua prática.

Palavras-Chave: assoalho pélvico, esportes, atletas, fisioterapia.

\section{Abstract}

Objectives: a) To identify the prevalence of urinary incontinence in athletes and practitioners of physical activity; b) To verify the impact of physical activity on athlete's pelvic floor muscles; and c) To investigate the scientific evidence about the physiotherapeutic treatment of urinary incontinence in athletes and practitioners of physical activity. Methods: A critical review of the literature was performed with searches in Pubmed and Lilacs databases, in the last ten years. We included experimental studies that evaluated the prevalence of urinary incontinence during sports practice, studies about the impact of physical activity on pelvic floor muscles, and intervention studies on pelvic floor dysfunction in athletes. Results: We included 17 studies. The prevalence of urinary incontinence ranged from $5 \%$ to $88.9 \%$ depending on the type of physical activity. The mean age of women was 24.9 years of age and the majority were nulligest. Regarding the impact of physical activity on the pelvic floor muscles of athletes, the main methods of muscle evaluation used were vaginal palpation, electromyography and three-dimensional ultrasonography. Finally, regarding the treatment, three intervention studies were found before and after, where different types of protocols were used with the aim of rehabilitating the pelvic floor muscles in female athletes with urinary incontinence, showing effectiveness. Conclusions: Muscle rehabilitation seemed to be effective in improving urinary incontinence, more randomized studies are needed to better prove the practice.
\end{abstract}

Keywords: pelvic floor, sports, athletes, physical therapy.
Afiliação dos autores

${ }^{1}$ Departamento de Fisioterapia, Faculdade de Educação Física e Fisioterapia, Universidade Federal de Uberlândia, Uberlândia; Minas Gerais, Brasil.

${ }^{2}$ Departamento de Ginecologia da Universidade Federal de São Paulo, Setor de Ginecologia do Esporte,

São Paulo; São Paulo, Brasil.

${ }^{3}$ Departamento de Fisioterapia, Faculdade de Educação Física e Fisioterapia, Programa de PósGraduação em Fisioterapia, Universidade Federal de Uberlândia, Uberlândia; Minas Gerais, Brasil.

\section{${ }^{*}$ Autor correspondente}

Laboratório de Desempenho Cinesiofuncional Pélvico e Saúde da Mulher (LADEP), Universidade

Federal de Uberlândia,

Rua Benjamin Constant, 1286, CEP: 38400-678, Uberlândia, Minas Gerais, Brasil e-mail: fisiorafaelamelo@gmail.com

Conflito de interesses

Os autores declararam não haver conflito de interesses.

Processo de arbitragem

Recebido: 30/12/2018 Aprovado: 06/03/2019 


\section{Introdução}

Diversos fatores podem influenciar o funcionamento correto dos músculos do assoalho pélvico (MAP) e a prática de exercícios físicos destaca-se entre eles ${ }^{1,2}$. A atividade física oferece muitos benefícios para a saúde, contudo, alguns autores afirmam que quando praticada de forma exorbitante, pode levar a disfunções tais como a incontinência urinária (IU) ${ }^{1,2}$.

A Sociedade Internacional de Continência (ICS) define a IU como qualquer perda involuntária de urina e pode ser dividida em três tipos: de urgência, de esforço e mista ${ }^{3}$. A Incontinência Urinária de Urgência (IUU) é caracterizada pelo forte desejo e repentino de urinar enquanto a Incontinência Urinária de Esforço (IUE) está relacionada com a perda de urina durante algum esforço que aumente a pressão abdominal, como tossir, espirrar, ocorrendo falha do esfíncter da uretra, e a Incontinência Urinária Mista (IUM) apresenta características tanto da IUE quanto da IUU ${ }^{3}$.

Diversos autores propuseram que os fatores que levam ao desenvolvimento da IU nas praticantes de atividade física são: lesões dos MAP que geram hipermobilidade da bexiga, alterações no posicionamento da uretra e o colo vesical, diminuindo a capacidade de fechamento durante o aumento da pressão abdominal $^{2}$, e a fadiga muscular, que é causada pela diminuição do suprimento sanguíneo, ocorrendo assim a diminuição da nutrição das fibras do tipo I que necessitam de oxigênio para uma boa contração ${ }^{4,5}$. Além desses fatores, a atividade muscular prolongada parece levar à diminuição da eficiência da contração, devido à diminuição da transmissão de sinal na junção neuromuscular ${ }^{1,5}$.

A IU pode ocorrer, ainda, devido à deficiência hormonal, causada por atividade física exaustiva, que pode levar à amenorreia de origem hipotalâmica, oligomenorréia ou liberação de opióides endógenos que vão levar à diminuição dos níveis de estrógeno, os quais possuem receptores no músculo detrusor que, ao se ligarem ao hormônio, contribuirão para a função de continência urinária sobre a uretra ${ }^{4,5}$. Outra causa citada é o aumento da pressão intra-abdominal que ocorre durante grande parte da prática esportiva, que pode ser maior que a pressão vesical e uretral, ocorrendo assim a perda de urina ${ }^{1}$.

Estudos suportam a teoria de que o surgimento da IU é mais comum em atletas que praticam esportes de alto impacto, como vôlei, basquete, trampolim, corrida, paraquedismo, atletismo, esportes de combates como o karatê e o judô, ginástica, equitação e fisiculturismo. Acredita-se que o impacto causado por essas atividades sobrecarregue os MAP feminino e leve a disfunções, principalmente à perda involuntária de urina, que pode causar alterações psicológicas e emocionais, muitas vezes levando a desistência da prática esportiva ${ }^{1,2,6}$

A literatura ainda é escassa acerca dos efeitos da atividade física exaustiva de alto impacto sobre os MAP e sua ligação com a IU. Ainda, faltam evidências do tratamento das disfunções dos MAP em atletas e praticantes de atividade física. Desta forma, este estudo tem três objetivos: a) Identificar a prevalência da IU em atletase praticantes de atividade física; b) Verificar o impacto da atividade física nos MAP de atletas; e c) Averiguar as evidências científicas sobre o tratamento fisioterapêutico da IU em atletas e praticantes de atividade física.

\section{Métodos}

Foi realizada uma revisão crítica da literatura na base de dados Publicações Médicas (PubMed) e Literatura LatinoAmericana e do Caribe em Ciências da Saúde (LILACS), entre os anos de 2008 a 2018. Os descritores utilizados foram: "pelvicfloor", "sports", "athletes", "physical therapy", sendo a busca feita com os quatro descritores combinados.

Os filtros utilizados foram: pesquisas dos últimos dez anos; em seres humanos; que envolvessem algum tipo de avaliação da prevalência de IU ou da condição dos MAP de atletas ou praticantes de atividade física ou que propusessem algum tipo de tratamento para mulheres atletas ou praticantes de atividade física.

Foram incluídos estudos nos idiomas português e inglês. Os critérios de inclusão para selecionar as publicações foram: estudos experimentais que avaliaram a prevalência de IU durante a prática esportiva ou o impacto da atividade física nos MAP, bem como estudos de intervenção que objetivaram tratar as disfunções dos MAP em praticantes de atividade física.
O Fluxograma 1 representa o processo de busca e seleção dos artigos que foram incluídos no estudo.

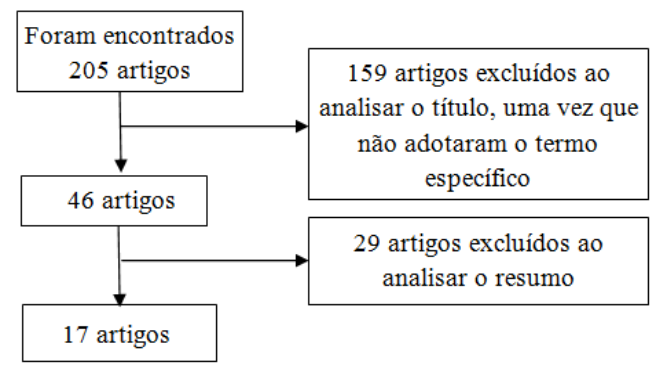

Figura 1. Fluxograma da seleção dos estudos para esta revisão. (Pesquisa realizada dia 26/11/2018).

\section{Resultados}

No que se refere à prevalência da IU em praticantes de atividade física, foram encontrados oito estudos. As informações foram sistematizadas e estão incluídas no Quadro 1. A prevalência da IU nos diversos estudos variou entre $5 \%$ e $88,9 \%$, a depender do tipo de atividade física. A idade média das mulheres que fizeram parte das amostras dos estudos selecionados foi de 24,9 anos e a maioria das mulheres era nuligesta.

No que tange o impacto da atividade física nos MAP de atletas e praticantes de atividade física, foram encontrados seis estudos, que foram descritos no Quadro 2. Os principais métodos de avaliação muscular utilizados pelos autores dos estudos incluídos foram: exame digital (palpação vaginal graduada pela Escala de Oxford), eletromiografia (EMG) e ultrassonografia tridimensional (US3D).

Quanto ao tratamento das disfunções dos MAP, não foram encontrados estudos randomizados controlados para incluir no presente estudo. Três estudos de intervenção tipo antes e depois foram encontrados e estão descritos no Quadro 3. Nesses estudos, diferentes tipos de protocolos foram utilizados com objetivo de reabilitação dos MAP em mulheres com queixa de IU e as intervenções estão detalhadas no Quadro 3.

\section{Discussão}

\section{Prevalência de IU}

Recentemente tem sido descrito nos estudos a IU em mulheres relacionada ao esporte $e^{10,11,23,24}$.A prevalência de IU em atletas está associada com o tipo de esporte, sendo que o acometimento é mais comum em praticantes de esportes de alto impacto $^{6,1}$. É importante ressaltar que trata-se de uma situação que afeta o componente psicológico da atleta, podendo levar ao abandono do esporte e consequente inatividade, tanto da atleta competitiva como da atleta recreacional ${ }^{14,21,25}$

É possível encontrar na literatura vários tipos de práticas esportivas que foram associados à IU sendo elas a ginástica artística, corrida, natação, hidroginástica, basquete, handebol, musculação, trampolim, voleibol, entre outros ${ }^{10,14,26-29}$. A perda involuntária de urina nas atletas que estão sujeitas a grandes impactos pode ser atribuída ao esforço exercido sobre a região abdominal, já que estas atividades levam a um aumento significativo da pressão intra-abdominal.

A IUE é comum entre os praticantes de atividade física. Larsen et al. ${ }^{5}$ relataram que $43 \%$ das 144 militares americanas incluídas em seu estudo apresentaram IUE e, além disso, 28,5\% apresentaram IUU e $28,5 \%$ IUM. Ainda, foi relatado em outro estudo que $50 \%$ das incontinentes se queixaram de perda durante os esforços, $41,7 \%$ durante a urgência e 1 voluntária apresentou IUM ${ }^{14}$. Para corroborar esses dados, Almeida et al. ${ }^{10}$ concluíram que o tipo mais prevalente de IU em atletas foi a de esforço. Analisando estes resultados, é possível notar que o tipo de incontinência com maior prevalência dentre as atletas é a IUE, que está relacionada a lesões dos MAP podendo gerar hipermobilidade da bexiga, mudanças na posição uretral e vesical ou fraqueza muscular. 
Quadro 1

Síntese dos estudos sobre prevalência de disfunções dos MAP em atletas.

\begin{tabular}{|c|c|c|c|}
\hline Autores & Características da amostra & Método utilizado para avaliar a Prevalência & Principais resultados \\
\hline Silva et al., $2018^{7}$ & $\begin{array}{l}\text { - Grupo 1: } 7 \text { mulheres com } \\
\text { disfunção urinária; } \\
\text { - Grupo 2: } 49 \text { mulheres sem } \\
\text { disfunção urinária. }\end{array}$ & $\begin{array}{l}\text { - Anamnese incluindo informações relacionadas à } \\
\text { frequência da atividade física; } \\
\text { - QuestionárioProtection, } \\
\text { Amount, Frequency, Adjustment, Body image. }\end{array}$ & $\begin{array}{l}\text { - } 100 \% \text { das mulheres do G1 e } 98 \% \text { das } \\
\text { mulheres do G2 praticavam musculação; } \\
-71,4 \% \text { das mulheres do } \mathrm{G} 1 \text { e } 53,1 \% \text { das } \\
\text { mulheres do G2 praticavam exercícios } \\
\text { aeróbicos; } \\
-12 \% \text { das mulheres apresentaram IU com } \\
\text { severidade de moderada a grave e sem } \\
\text { associação com idade, modalidade, tempo } \\
\text { de prática, turno e frequência de } \\
\text { exercícios. }\end{array}$ \\
\hline Hagovska et al., $2017^{8}$ & - 278 mulheres atletas nulíparas. & $\begin{array}{l}\text { - Questionário ICIQ-SF; } \\
\text { - Questionário OveractiveBladder; } \\
\text { - Escala Urinary Incontinence Quality of Life; } \\
\text { - Questionário International Physical Activity. }\end{array}$ & $\begin{array}{l}\text { - A prevalência de IUE nas atletas foi de } \\
19,6 \% \text { no vôlei, } 15,6 \% \text { em mulheres que } \\
\text { frequentam academia, } 23,8 \% \text { no atletismo, } \\
14,7 \% \text { no basquete, } 16,6 \% \text { no handebol, } \\
6,9 \% \text { na dança e } 5 \% \text { no futebol. }\end{array}$ \\
\hline Alves et al., $2017^{9}$ & $\begin{array}{l}\text { - Atletas de esportes com alto } \\
\text { impacto: } 136 \text {; } \\
\text { - Atletas de esportes de baixo } \\
\text { impacto: } 109 \text {. }\end{array}$ & $\begin{array}{l}\text { - Questionário sociodemográfico; } \\
\text { - Questionário ICIQ; } \\
\text { - Questionário Kings Health. }\end{array}$ & $\begin{array}{l}\text { - 22,9\% das mulheres relataram sintomas } \\
\text { de IU com ocorrência pelo menos mensal; } \\
\text { - } 60,7 \% \text { relataram IUE, } 25 \% \text { relataram IUU } \\
\text { e 14,3\% relataram IUM; } \\
\text { - As mulheres praticantes de esporte de } \\
\text { alto impacto relataram maior frequência na } \\
\text { IU; } \\
\text { - Independentemente da intensidade do } \\
\text { esporte, o volume de atividade física } \\
\text { apresentou associação positiva com a } \\
\text { frequência de perda de urina. }\end{array}$ \\
\hline Almeida et al., $2015^{10}$ & $\begin{array}{l}\text { - } 96 \text { não atletas; } \\
\text { - } 67 \text { atletas. }\end{array}$ & $\begin{array}{l}\text { - Questionário com informações relacionadas ao } \\
\text { estado de saúde, dados demográficos e pessoais, } \\
\text { características da prática desportiva e sintomas } \\
\text { relacionados ao AP (IU, incontinência anal, } \\
\text { dispareunia, prolapso de órgãos pélvicos, etc). }\end{array}$ & $\begin{array}{l}\text { - A IU foi queixa de } 52,2 \% \text { mulheres no } \\
\text { grupo atletas e } 27,1 \% \text { mulheres no grupo } \\
\text { não atletas; } \\
\text { - Houve maior proporção de IU entre os } \\
\text { atletas que praticaram ginástica artística e } \\
\text { trampolim }(88,9 \%) \text {, seguido de natação } \\
\text { (50,0\%), judo }(44,4 \%) \text {, e voleibol }(43,5 \%) \text {; } \\
\text { - O tipo mais prevalente de IU foi a IUE na } \\
\text { ginástica artística e atletas de trampolim } \\
\text { ( } 87,5 \%) \text {, seguidos por natação }(84,6 \%) \text { e } \\
\text { atletas de judô }(75,0 \%) \text {. }\end{array}$ \\
\hline Roza et al., $2015^{11}$ & $\begin{array}{l}\text { - } 22 \text { atletas de elite ou atletas } \\
\text { não-elite. }\end{array}$ & $\begin{array}{l}\text { - Questionário com informações relacionadas a } \\
\text { características pessoais com variáveis } \\
\text { demográficas e antropométricas, atividade } \\
\text { esportiva, e avaliação dos sintomas urinários; } \\
\text { - Questionário ICIQ-UI-SF. }\end{array}$ & $\begin{array}{l}\text { - } 72,7 \% \text { relataram IU durante a prática de } \\
\text { trampolim e dentre essas } 3 \text { relataram IU } \\
\text { durante tosses e espirros; } \\
\text { - Todas relataram que as perdas urinárias } \\
\text { começaram após a prática com trampolim; } \\
\text { - } 68,8 \% \text { das mulheres relataram que a IU } \\
\text { não interfere ou interfere suavemente na } \\
\text { qualidade de vida. }\end{array}$ \\
\hline Poswiata et al., $2014^{12}$ & $\begin{array}{l}\text { - } 57 \text { esquiadoras; } \\
\text { - } 55 \text { corredoras. }\end{array}$ & $\begin{array}{l}\text { - Questionário anônimo autoaplicável; } \\
\text { - Questionário Urogenital Distress Inventory - SF. }\end{array}$ & $\begin{array}{l}\text { - } 50 \% \text { das atletas perdem pequenas } \\
\text { quantidades de urina; } \\
-45,54 \% \text { apresentaram IUE, } 27,68 \% \text { IUU } \\
\text { E } 18,75 \% \text { IUM. }\end{array}$ \\
\hline Vitton et al., $2011^{13}$ & $\begin{array}{l}\text { - Grupo de esporte intenso: } 169 \\
\text { mulheres; } \\
\text { - Grupo de esporte não-intenso: } \\
224 \text { mulheres. }\end{array}$ & $\begin{array}{l}\text { - Questionário anônimo autoaplicável (dados } \\
\text { demográficos, saúde pessoal, características do } \\
\text { esporte, informações sobre sintomas relacionados } \\
\text { aos MAP). }\end{array}$ & $\begin{array}{l}\text { - O esporte de alto nível é um fator de } \\
\text { risco significativo para incontinência anal; } \\
\text { - A prevalência de incontinência anal foi } \\
\text { estatisticamente maior no grupo de } \\
\text { esporte intenso ( } 14,8 \%) \text { quando } \\
\text { comparado ao grupo de esporte não- } \\
\text { intenso }(4,9 \%) ; \\
\text { - A prevalência de IU foi de } 33,1 \% \text { no } \\
\text { grupo de esporte intenso e de } 18,3 \% \\
\text { grupo de esporte não-intenso; } \\
\text { - } 61,8 \% \text { das mulheres observaram a IU } \\
\text { apenas durante a prática esportiva. }\end{array}$ \\
\hline Santos et al., $2009^{14}$ & $\begin{array}{l}\text { - } 58 \text { mulheres nulíparas } \\
\text { estudantes de Educação Física. }\end{array}$ & $\begin{array}{l}\text { - Questionário com informações relacionadas aos } \\
\text { dados sociodemográficos, sintomatologia da IU, } \\
\text { práticas esportivas, e influência da IU na vida das } \\
\text { estudantes. }\end{array}$ & $\begin{array}{l}-20,7 \% \text { relataram perda de urina, destas, } \\
75 \% \text { durante atividades esportivas e } 25 \% \\
\text { fora das atividades; } \\
\text { - As modalidade esportivas mais } \\
\text { relacionadas com a perda urinária foram } \\
\text { natação }(33,3 \%) \text { e cama elástica }(22,2 \%) \text {; } \\
-50 \% \text { das mulheres relataram IUE, } 41,7 \% \\
\text { das mulheres relataram IUU e uma mulher } \\
\text { relatou IUM. }\end{array}$ \\
\hline
\end{tabular}
Incontinência Urinária de Esforço; IUU: Incontinência Urinária de Urgência; IUM: Incontinência Urinária Mista.

A prevalência de IU frequentemente está associada à idade e aquelas mulheres que já passaram pela experiência da gestação e do parto. Porém, muitos estudos recentes mostraram que mulheres jovens e nulíparas também estão sujeitas a apresentar $I U^{25}$. Os estudos selecionados para esta revisão reforçam essa informação, visto que a média de idade das mulheres estudadas foi 24,9 anos e a maioria das mulheres era nuligesta. Tal fato reforça a hipótese de que as atividades de alto impacto podem levar adisfunções dos MAP.

Alguns estudos compararam a prevalência de IU entre atletas e não atletas, e concluiram que atletas de elite têm alta prevalência de IU quando comparadas a um grupo controle, e ainda que, na prática de esportes de alto impacto, o risco aumenta ${ }^{12,23,27,30-32}$. Já Salvatore et al. ${ }^{33}$, ao analisar atletas recreacionais, observaram que a incidência de IU entre as mulheres era menor, e concluíram que o impacto da incontinência urinária nas atletas recreacionais não é tão significativo quando comparado com a prática esportiva profissional. Sugere-se que a prevalência maior de IU nas atletas de elite pode ser atribuída não somente ao impacto a que estas atletas estão sujeitas, mas principalmente devido àrotina, frequência, duração e intensidade dos treinamentos, que, na maioria das vezes, são exaustivos. 
Quadro 2

Síntese dos estudos sobre tratamento da incontinência urinária em atletas.

\begin{tabular}{|c|c|c|c|}
\hline Autores & $\begin{array}{l}\text { Características da } \\
\text { amostra }\end{array}$ & Método de Avaliação AP & Principais Resultados \\
\hline $\begin{array}{l}\text { Wollmann et al., } \\
2018^{15}\end{array}$ & $\begin{array}{l}\text { - } 10 \text { mulheres praticantes de } \\
\text { futsal amador. }\end{array}$ & $\begin{array}{l}\text { - Exame físico com manometria } \\
\text { de pressão (perineometria) dos } \\
\text { MAP. }\end{array}$ & $\begin{array}{l}\text { - Em uma pontuação de } 0 \text { a } 100 \text { (Escala Sauer), as mulheres } \\
\text { apresentaram força dos MAP equivalente a } 24,4( \pm 11,7) \text {, } \\
\text { considerada musculatura regular, de acordo com a interpretação da } \\
\text { Escala; } \\
\text { - O tempo de contrações dos MAP das atletas foi de } 9,2( \pm 1,6) \\
\text { segundos; } \\
\text { - Quanto maior o tempo que as atletas jogam, menor é o tempo de } \\
\text { contração da musculatura. }\end{array}$ \\
\hline $\begin{array}{l}\text { Leitner et al., } \\
2017^{16}\end{array}$ & $\begin{array}{l}\text { - Incontinentes: } 22 \text { mulheres; } \\
\text { - Continentes: } 28 \text { mulheres. }\end{array}$ & $\begin{array}{l}\text { - EMG dos MAP durante corrida } \\
\text { na esteira antes e após o } \\
\text { impacto da aterrisagem na } \\
\text { esteira em três velocidades. }\end{array}$ & $\begin{array}{l}\text { - A pré-atividade média de EMG e a atividade reflexa aumentaram } \\
\text { significativamente com a velocidade; } \\
\text { - Para todos os intervalos de tempo e para todas as velocidades, os } \\
\text { MAP estavam sempre ativos durante a corrida; } \\
\text { - A corrida parece desencadear a pré-ativação antes e ativação } \\
\text { reflexa após o toque de calcanhar, sem diferenças estatísticas entre } \\
\text { os grupos. }\end{array}$ \\
\hline $\begin{array}{l}\text { Araújo et al., } \\
2015^{17}\end{array}$ & $\begin{array}{l}\text { - Grupo caso: } 49 \text { atletas } \\
\text { nulíparas praticantes de } \\
\text { esporte de alto rendimento e } \\
\text { alto impacto; } \\
\text { - Grupo controle: } 44 \text { nulíparas } \\
\text { sedentárias. }\end{array}$ & $\begin{array}{l}\text { - Avaliação funcional dos MAP } \\
\text { foi realizada pelo exame digital } \\
\text { (graduada pela AFA) e } \\
\text { manometria de pressão } \\
\text { (perineometria). }\end{array}$ & $\begin{array}{l}\text { - A maioria das atletas apresentou AFA superior a } 3 \text { e nenhuma } \\
\text { sedentária apresentou AFA } 5, \text { com diferença estatisticamente } \\
\text { significante; } \\
\text { - Não houve diferença entre os grupos consoante àpressão vaginal } \\
\text { de repouso; } \\
\text { - As atletas apresentaram maiores valores de pressão vaginal } \\
\text { máxima }(\mathrm{cmH} 2 \mathrm{O})(70,1( \pm 2,4)) \text { quando comparadas às sedentárias } \\
(34,3( \pm 1,7)) \text { e este resultado foi estatisticamente significante } \\
(\mathrm{p}<0,001) ; \\
\text { - As atletas praticantes de basquete tiveram os maiores valores da } \\
\text { pressão vaginal máxima }(77,2 \mathrm{cmH} 2 \mathrm{O}) \text { quando comparadas as } \\
\text { ginastas }(65,5 \mathrm{cmH} 2 \mathrm{O}) \text { e corredoras }(65,4 \mathrm{cmH} 2 \mathrm{O}) \text {, sem diferença } \\
\text { estatisticamente significante; } \\
\text { - A prevalência de incontinência urinária nas atletas foi de } 76 \% \text { e } \\
\text { somente } 16 \% \text { nas sedentárias; } \\
\text { - As atletas incontinentes, tiveram valores elevados de pressão } \\
\text { vaginal máxima quando comparado as sedentárias incontinentes. }\end{array}$ \\
\hline $\begin{array}{l}\text { Luginbuehl et al., } \\
2015^{18}\end{array}$ & $\begin{array}{l}\text { - } 10 \text { mulheres nulíparas } \\
\text { continentes. }\end{array}$ & $\begin{array}{l}\text { - EMG dos MAP durante corrida } \\
\text { na esteira em três velocidades. }\end{array}$ & $\begin{array}{l}\text { - Todas as variáveis de EMG dos MAP de todas as velocidades da } \\
\text { corrida mostraram valores mais altos quando comparados com a } \\
\text { atividade dos MAP durante a postura em pé, sem contração } \\
\text { voluntária; } \\
\text { - Os resultados sugerem que existe uma pré-atividade dos MAP e } \\
\text { atividade reflexa durante a corrida. }\end{array}$ \\
\hline $\begin{array}{l}\text { Junior et al., } \\
2014^{19}\end{array}$ & $\begin{array}{l}\text { - } 24 \text { mulheres corredoras }(11 \\
\text { correm }<25 \mathrm{~km} \text { por semana e } \\
13 \text { correm }>25 \mathrm{~km} \text { por } \\
\text { semana). }\end{array}$ & $\begin{array}{l}\text { - EMG dos MAP; } \\
\text { - US3D. }\end{array}$ & $\begin{array}{l}\text { - A distância semanal percorrida não apresentou correlação } \\
\text { significativa com nenhum dos parâmetros relacionados à } \\
\text { eletromiografia dos MAP ou US3D; } \\
\text { - As correlações entre a EMG e a US3D mostraram diferenças } \\
\text { significativas apenas em relação à EMG em repouso e à espessura } \\
\text { do levantador do ânus durante a manobra de Valsalva ( } r=0,46 ; \\
p=0,04) ; \text { na variação da EMG observada entre contração lenta e } \\
\text { repouso, e na variação na espessura do levantador entre Valsalva e } \\
\text { repouso }(r=-0,46 ; p=0,04) ; \\
\text { - Não houve diferenças funcionais e anatômicas entre as que } \\
\text { percorriam > } 25 \text { km por semana, mesmo sendo mais velhas, em } \\
\text { comparação com as que corriam }<25 \text { km por semana. }\end{array}$ \\
\hline $\begin{array}{l}\text { Reis et al., } \\
2011^{20}\end{array}$ & $\begin{array}{l}\text { - } 10 \text { atletas de basquetebol; } \\
\text { - } 10 \text { atletas de voleibol. }\end{array}$ & $\begin{array}{l}\text { - Exame digital (graduado pela } \\
\text { AFA); } \\
\text { - Avaliação da capacidade de } \\
\text { contração dos MAP por meio do } \\
\text { Biofeedback EMG Fênix. }\end{array}$ & $\begin{array}{l}\text { - As atletas de basquetebol apresentaram média de AFA } 3( \pm 0,5) \text { e } \\
\text { as atletas de voleibol apresentaram média de AFA } 4( \pm 0,5), \text { sem } \\
\text { diferença estatística; } \\
\text { - Na avaliação da capacidade de contração dos MAP das atletas } \\
\text { com o Biofeedback EMG, a modalidade de basquetebol obteve } \\
\text { média de } 21( \pm 1,5), \text { na modalidade de voleibol a média foi de } \\
20( \pm 0,8) \text {, sem diferença estatística; } \\
\text { - Não foram encontradas diferenças entre os resultados obtidos nos } \\
\text { níveis de avaliação funcional do AP e pelo Biofeedback EMG em } \\
\text { relação à modalidade esportiva. }\end{array}$ \\
\hline
\end{tabular}

MAP: Músculos do Assoalho Pélvico; AFA: Avaliação Funcional dos MAP; EMG: eletromiografia; US3D: Ultrassonografia Tridimensional.

\section{Impacto da atividade física sobre os MAP}

Nos diversos estudos incluídos, os métodos de avaliação da musculatura do AP foram a palpação digital, a eletromiografia

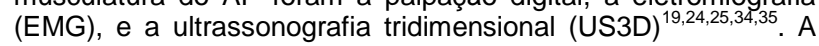
palpação digital avalia a contração dos MAP por meio da escala de Oxford, que detecta desde a ausência de contração até uma forte contração ${ }^{24}$. A EMG tem sido muito utilizada para avaliar a atividade elétrica gerada pela contração muscular do AP em mulheres com IU e prolapso genital ${ }^{36}$, é um método utilizado e bem estabelecido para mensurar a atividade voluntária e involuntária dos músculos esqueléticos ${ }^{37}$ avaliando, portanto, a atividade elétrica gerada pela contração do músculo, que não necessariamente indica a força muscular. Ainda, como método de avaliação, foi utilizada a US3D, uma técnica não invasiva utilizada para avaliar os MAP e vem sendo cada vez mais utilizadapara avaliar o pavimento pélvico e para diagnosticar os efeitos de gestação, parto e impacto causado por esportes ${ }^{18}$, fornecendo informações anatômicas sobre a posição, espessura e tamanho do músculo ${ }^{38}$.

Ao analisar a morfologia, observou-se que os MAP de mulheres atletas são mais espessos quando comparados aos de mulheres não atletas ${ }^{19,34}$. Roza et al. ${ }^{24}$, ao avaliarem a musculatura pubovisceral do AP por meio da RM, demonstraram que em nível anterior há um aumento da espessura da musculatura dos MAP em atletas incontinentes comparados às continentes. O músculo pubovisceral é mais espesso em atletas incontinentes e os autores atribuíram o achado a uma tentativa do músculo de neutralizar a diminuição da capacidade de resposta do esfíncter uretral, já que o músculo pubovisceral e o esfincter uretral fecham a uretra durante o aumento da pressão intra-abdominal para impedir a micção ${ }^{39}$.

Em contrapartida, em outro estudo foi utilizada a EMG e o US3D para avaliar a atividade elétrica dos MAP de corredoras divididas em dois grupos (um grupo de mulheres que percorriam $>25 \mathrm{~km}$ e outro grupo que percorria $<25 \mathrm{~km}$ ) e não foi observada qualquer diferença entre os dois grupos principalmente em relação à atividade elétrica, espessura e lesões do músculo pubovisceral. Foi observado também que a contração voluntária máxima dos MAP das corredoras apresentou boa atividade elétrica, inferindo indiretamente que a função muscular provavelmente está adequada ${ }^{19}$. Todavia, é importante ressaltar que não foram incluídas atletas de elite, mas corredoras recreacionais e muitas delas já haviam passado pelo ciclo-gravídico puerperal, o que pode ter influenciado nos resultados. 
Quadro 3

Síntese dos estudos sobre prevalência de disfunções dos MAP em atletas.

\begin{tabular}{|c|c|c|c|c|}
\hline Autores & Características da amostra & Desfechos avaliação & Protocolo de Intervenção & Principais Resultados \\
\hline $\begin{array}{l}\text { Ferreira et al., } \\
2014^{2}\end{array}$ & $\begin{array}{l}\text { - Mulheres jogadoras de } \\
\text { vôlei, nulíparas, } \\
\text { apresentando IUE; } \\
\text { - Grupo Experimental: } 16 \\
\text { mulheres; } \\
\text { - Grupo Controle: } 16 \\
\text { mulheres. }\end{array}$ & $\begin{array}{l}\text { - Questionário; } \\
\text { - Teste do absorvente; } \\
\text { - Diário miccional. }\end{array}$ & $\begin{array}{l}\text { - Grupo Experimental: programa de } \\
\text { reabilitação dos MAP (ação } \\
\text { educativa, consciência dos MAP, } \\
\text { folheto informativo). } \\
\text { - Programa de reabilitação: } 30 \\
\text { contrações sustentadas e } 4 \\
\text { contrações rápidas após cada } \\
\text { contração sustentada, em posições } \\
\text { diferentes, diariamente, durante } \\
\text { três meses; } \\
\text { - Grupo Controle: folheto } \\
\text { informativo }\end{array}$ & $\begin{array}{l}\text { - O maior percentual de perda urinária } \\
\text { foi desencadeado durante o } \\
\text { treinamento, em } 87,5 \% \text { das mulheres do } \\
\text { GE e em } 81,3 \% \text { das mulheres no GC; } \\
\text { - Após três meses de intervenção, o GE } \\
\text { reduziu significativamente a ocorrência } \\
\text { de perda urinária, com queda de } 45,5 \% \\
\text { em comparação com } 4,9 \% \text { no GC, com } \\
\text { diferença estatisticamente significante; } \\
\text { - A frequência das perdas de urina } \\
\text { reduziram } 14,3 \% \text { no GE e } 1,9 \% \text { no GC. }\end{array}$ \\
\hline $\begin{array}{l}\text { Roza et al., } \\
2012^{21}\end{array}$ & $\begin{array}{l}\text { - } 7 \text { mulheres estudantes } \\
\text { nulíparas praticantes de } \\
\text { altos níveis de atividade } \\
\text { física. }\end{array}$ & $\begin{array}{l}\text { - Questionário } \\
\text { sociodemográfico; } \\
\text { - Questionário IPAQ-SF; } \\
\text { - Questionário ICIQ-SF; } \\
\text { - Exame digital } \\
\text { (graduado pela Escala } \\
\text { de Oxford); } \\
\text { - Manometria de pressão } \\
\text { (perineometria). }\end{array}$ & $\begin{array}{l}\text { - Programa de reabilitação dividido } \\
\text { em quatro etapas, sendo cada } \\
\text { etapa com duração de } 2 \text { semanas: } \\
\text { 1) Conscientização dos MAP com } \\
\text { base no feedback durante a } \\
\text { palpação vaginal; } 2 \text { ) Contrações } \\
\text { dos MAP em diferentes posições } \\
\text { com pesos progressivos; 3) } \\
\text { Tentativas de contrair os MAP } \\
\text { durante corridas e caminhadas; 4) } \\
\text { Tentativas de contrair os MAP } \\
\text { durante atividades esportivas. }\end{array}$ & $\begin{array}{l}\text { - Foi observada melhora } \\
\text { estatisticamente significativa na pressão } \\
\text { de repouso vaginal (antes: } 38,4( \pm 15,7) \text { - } \\
\text { depois: } 55,8( \pm 9,00)) \text { e na contração } \\
\text { voluntária máxima (antes: } 73,4( \pm 24,9) \text { - } \\
\text { depois: } 89,8( \pm 19,1)) \text { após o período de } \\
\text { treinamento de } 8 \text { semanas; } \\
\text { - Foram encontradas diferenças } \\
\text { estatisticamente significativas antes e } \\
\text { após a intervenções em relação à } \\
\text { frequência de perda (antes: } 1,6( \pm 1,5)- \\
\text { depois: } 0,1( \pm 0,4)) \text {; quantidade de perda } \\
\text { (antes: } 2,6( \pm 1,5)-\text { depois: } 0,3( \pm 0,8)) ; \text { e } \\
\text { escore do ICIQ-SF (antes: } 4,08( \pm 6,0)- \\
\text { depois: } 0,75( \pm 2,6)) \text {. } \\
\text { - } 6 \text { das } 7 \text { atletas apresentaram resolução } \\
\text { completa do quadro. }\end{array}$ \\
\hline $\begin{array}{l}\text { Massimo et al., } \\
2010^{22}\end{array}$ & $\begin{array}{l}\text { - } 3 \text { mulheres atletas } \\
\text { nulíparas jogadoras de } \\
\text { vôlei. }\end{array}$ & $\begin{array}{l}\text { - Diário Miccional; } \\
\text { - Exame urodinâmico; } \\
\text { - Avaliação } \\
\text { uroginecológica; } \\
\text { - Teste Puborretal } \\
\text { graduado pela Escala de } \\
\text { Oxford }\end{array}$ & $\begin{array}{l}\text { Programa de reabilitação dividido } \\
\text { em } 4 \text { partes: } 1 \text { ) Estimulação } \\
\text { elétrica funcional (FES) durante } 20 \\
\text { minutos, uma vez por semana } \\
\text { durante } 3 \text { meses; } 2 \text { ) } \\
\text { Biofeedbackdurante } 15 \text { minutos, } \\
\text { uma vez por semana durante } 3 \\
\text { meses; } 3 \text { ) Exercícios para os MAP; } \\
\text { 4) Exercícios para MAP utilizando } \\
\text { cones vaginais (mantendo por } 1 \\
\text { minuto no início e tentando } \\
\text { alcançar } 10 \text { minutos e durante os } \\
\text { esforços e atividades). }\end{array}$ & $\begin{array}{l}\text { - Todas as atletas apresentaram } \\
\text { melhora no Teste Puborretal graduado } \\
\text { pela Escala de Oxford (Atleta 1: 2-5; } \\
\text { Atleta 2: 1-5; Atleta 3: 2-5); } \\
\text { - Após o programa combinado de } \\
\text { reabilitação dos MAP as atletas não } \\
\text { tiveram perda de urina durante atividade } \\
\text { esportiva, atividades fitness ou atividade } \\
\text { de vida diária; } \\
\text { - Após reabilitação não houve } \\
\text { necessidade de utilizar absorventes. }\end{array}$ \\
\hline
\end{tabular}

Outros pesquisadores utilizaram a EMG para avaliar os MAP em corredoras e notou-se que durante a corrida há um constante aumento da atividade elétrica desses músculos, podendo ter relação com a IU por fadiga muscular, devido ao uso excessivo da musculatura por constante aumento da pressão intra-abdominal, perdendo sua força para o controle da micção ${ }^{25}$.

O US3D/4D foi utilizado no estudo de Kruger et al ${ }^{40}$ para comparar os MAP de atletas de alto impacto com grupo controle.Foi observada uma série de diferenças funcionais e morfológicas nos MAP das atletas, dentre elas o aumento significativo do diâmetro do musculo levantador do ânus, que foi atribuída ao fato de que as atletas têm maior consciência cinestésica e são capazes de recrutar os MAP em atividades específicas, além de possuir maior resistência abdominal. Por fim, o grupo de atletas mostrou uma combinação de musculatura mais espessa e com maior distensibilidade quando comparadas ao grupo controle. Os autores propuseram que as diferenças observadas podem ter ocorrido devido às alterações biomecânicas do tecido conjuntivo ou musculares, que podem ser anteriores ou ser consequência do treinamento de alto impacto.

Ree et al. $^{35}$ recrutaram um grupo de 12 nuligestas que apresentavam sinais de IU durante a prática de exercícios extenuantes e observaram redução de $20 \%$ na pressão média da contração voluntária máxima após o exercício e um aumento de $2 \%$ após o período de descanso. O autor relaciona este achado com a fadiga muscular, que pode ser considerada um fator de alerta para sobrecarga muscular. Poucos estudos buscaram avaliar a fadiga muscular, contudo, estudos seguindo essa linha de pesquisa podem ser promissores, uma vez que a fadiga muscular de maneira geral acontece com a prática de atividades extenuantes e especula-se que não seria diferente para os MAP.

Outro ponto relevante é que a avaliação dos MAP em atletas e não atletas nos estudos incluídos, na maioria das vezes, foi feita em situações de repouso e não necessariamente capturam a real condição muscular durante longos períodos de treinamento. $\dot{E}$ importante ressaltar que, muitas vezes, as disfunções dos MAP dessas atletas não estão relacionadas somente com a fraqueza muscular, mas também com a fadiga decorrente da intensidade do exercício realizado. Finalmente, é importante ponderar que faltam evidências sobre como avaliar corretamente os MAP de atletas com o objetivo de descrever o impacto dos exercícios nesses músculos durante a prática esportiva. Além disso, são necessários estudos mais concisos que comparem os MAP de atletas e não atletas, bem como de atletas de elite e atletas recreacionais.

\section{Tratamento das disfunções dos MAP}

No que se refere ao tratamento de disfunções dos MAP, os autores avaliaram o efeito dos programas de reabilitação dos MAP em atletas. Foram selecionados três estudos e houve redução da frequência e da quantidade de perda urinária, bem como melhora dos sintomas clínicos associados à IU. Atualmente, a intervenção de primeira linha no tratamento da IUE tem sido os programas de treinamento dos MAP ${ }^{41}$.

No estudo de Roza et $\mathrm{al}^{21}$ para reabilitação dos MAP de estudantes atletas consideradas incontinentes, foi elaborado um programa de reabilitação que incluiu a conscientização dos MAP, contrações dos MAP em diferentes posições com pesos progressivos, tentativas de contrair os MAP durante corridas e caminhadas e tentativas de contrair os MAP durante atividades esportivas. Ao final, 6 das 7 atletas incluídas consideraram-se curadas. Em acordo com essa metodologia de treinamento, $\mathrm{B}^{42}$ aponta que cerca de um terço das mulheres com disfunção dos MAP não são capazes de contrair esses músculos corretamente, ou seja, a conscientização dos MAP por meio do exame digital torna-se etapa indispensável nos programas de reabilitação para que se tenha conhecimento se a atleta consegue realizar corretamente uma contração.

No estudo de Massimo et $\mathrm{al}^{22}$ foi proposto um programa de reabilitação que incluiu o uso do FES por 20 minutos por semana, do biofeedback por 15 minutos, ambos durante 3 meses, e exercícios para os MAP ativos-livres e com o uso de cones vaginais, em de forma domiciliar, após um acompanhamento preliminar com profissional. Toda a reabilitação durou 4 meses, tendo uma visita semanal e um diário miccional de 48 horas. Notou-se que, após a reabilitação, 
as atletas não apresentaram perda de urina durante a atividade esportiva ou na vida diária e, além disso, houve diminuição significativa do uso de absorventes. Também não houve nenhum efeito colateral ou complicações com a reabilitação.

Por fim, Ferreira et al. ${ }^{2}$ incluíram em seu estudo experimental 32 jogadoras de vôlei de um clube profissional de Portugal. 16 mulheres foram incluídas no grupo experimental que se submeteu a três meses de treinamento com protocolo e receberam folheto educativo. $O$ protocolo de treinamento consistiu em ação educativa, conscientização dos MAP e recebimento do folheto educativo. As atletas do grupo experimental foram incentivadas a realizar 30 contrações sustentadas e quatro contrações rápidas após cada contração sustentada, em diferentes posições e diariamente por três meses, em domicílio.Outras 16 mulheres receberam apenas 0 folheto educativo sobre exercícios para os MAP. Ao final, houve diminuição da perda urinária em $45,5 \%$ nas atletas do grupo experimental e de $4,9 \%$ nas atletas do grupo controle. Os autores concluíram que a intervenção com treinamento dos MAP foi eficaz.

As evidências aqui descritas são limitadas e devem ser avaliadas com cautela. Os estudos selecionados para esta revisão não são ensaios clínicos randomizados controlados, o que seria ideal. Não foram encontrados estudos com essa característica e isso revela a grande escassez de evidência sobre o tema científica sobre o tema. Portanto, essas se fazem sugestões para futuros estudos incluindo essa linha de pesquisa.

\section{Conclusão}

Há maior probabilidade de incontinência urinária em atletas de elite e 0 alto impacto das atividades parece ser a principal causa. As alterações dos MAP dessas atletas de elite e das praticantes de atividade física podem estar presentes, principalmente nos grupos de elite, devido àintensa rotina de treinamento, todavia, há necessidades de mais estudos sobre as condições desse grupo muscular antes, durante e após a atividade física para melhor conhecimento de como se comporta a musculatura diante dessas três situações. Com relação àreabilitação fisioterapêutica, o tratamento parece ser eficaz para a melhora da incontinência urinária. São necessários mais estudos randomizados para melhor comprovação da sua prática.

\section{Referências}

1. Barros JD, Lucena ACT, Anselmo CWSF. Incontinência urinária de esforço em atletas do sexo feminino: uma revisão da literatura. An. Fac. Med. Univ. Fed. Pernamb 2007; 52(2):173-180.

2. Ferreira S, Ferreira M, Carvalhais A, Santos PC, Rocha P, Brochado G. Reeducation of pelvicfloor muscles in volleyball athletes. RevAssocMedBras 2014; 60(5):428-433.

3. Haylen BT, de Ridder D, Freeman RM, Swift SE, Berghmans B, Lee J, Monga A, Petri E, Rizk DE, Sand PK, Schaer GN. An International Urogynecological Association (IUGA/International Continence Society (ICS) joint report on the terminology for female pelvic floor dysfunction. Neurol Urodyn 2010; 29(1):4-20.

4. Eliasson K, Edner A, Mattsson E. Urinary incontinence in very young and mostly nulliparous women with a history of regular organized high-impact trampoline training: occurrence and risk factors. Int Urogynecol J 2008; 19(5):687-96

5. Larsen WI, Yavorek TA. Pelvic organ prolapse and urinary incontinence in nulliparous women at the United States Military Academy. IntUrogynecol J PelvicFloorDysfunct 2006; 17(3):208-10.

6. Almeida MBA, Barra AA, Figueiro EM, Velloso FSB, Silva AL, Monteiro MVC, Rodrigues AM. Disfunções de assoalho pélvico em atletas. Femina 2011; 39(8):395-402.

7. Silva LB, Santos WO, Araujo NS, Rodrigues CNC, Nunes EFC. Disfunções urinárias em mulheres praticantes de atividade física em academias - um estudo transversal. RevPesqFisio 2018;8(1):71-8.

8. Hagovska M, Švihra J, Buková A, Dračková D, Švihrová V. Prevalence and risk of sport types to stress urinary incontinence in sportswomen: A crosssectional study. NeurourolUrodyn 2018; 37:1957-64.

9. Alves JO, Luz STD, Brandão S, Da Luz DM, Jorge RN, Da Roza T. Urinary Incontinence in Physically Active Young Women: Prevalence and Related Factors. Int J Sports Med 2017;38(12): 937-41.

10. Almeida MB, Barra AA, Saltiel F, Silva-Filho AL, Fonseca AM, Figueiredo EM. Urinary incontinence and other pelvic floor dysfunctions in female athletes in Brazil: A cross-sectional study. Scand J MedSci Sports 2016; 26(9):1109-16.

11. Da Roza T, Brandão S, Mascarenhas T, Jorge RN, Duarte JA. Volume of training and the ranking level are associated with the leakage of urine in young female trampolinists. Clin J Sport Med 2015; 25(3): 270-5.
12. Poswiata A, Socha T, Poara J. Prevalence of Stress Urinary Incontinence in Elite Female Endurance Athletes. J Hum Kinet 2004; 44:91-6.

13. Vitton V, Baumstarck-Barrau K, Brardjanian S, Caballe I, Bouvier M, Grimaud JC. Impact of high-level sport practice on anal incontinence in a healthy young female population. J Womens Health (Larchmt) 2011; healthy young
20(5):757-63.

14. Santos ES, Caetano AS, Tavares MCG, Lopes MHB. Incontinência urinária entre estudantes de educação física. Rev Esc Enferm USP 2009 43(2):307-12

15. Wollmann L, Goulart CL, Mansour KMK, Cerentini TM, Carvalho LL, Sudbrack AC. Influência da prática de futsal amador na força muscular do assoalho pélvico. Rev PesqFisio 2018; 8(3):313-8.

16. Leitner M, Moser H, Eichelberger P, Kuhn A, Radlinger L. Evaluation of pelvic floor muscle activity during running in continent and incontinent women: An exploratory study. NeurourolUrodyn 2017; 36(6):1570-6.

17. Araujo MP, Parmigiano TR, Negra LGD, Torelli L, Carvalho CG, Wo L, Manito ACA, Girão MJBC, Sartori MGF. Avaliação do assoalho pélvico de atletas: existe relação com a incontinência urinária? RevBrasMed Esporte 2015; $21(6): 442-6$

18. Luginbuehl H, Naeff R, Zahnd A, Baeyens JP, Kuhn A, Radlinger L. Pelvic floor muscle electromyography during different running speeds: an exploratory and reliability study. Arch GynecolObstet 2016; 293(1):117-24.

19. Junior EA, Bella ZIJD, Zanetti MRD, Araújo MP, Petricelli CD, Martins WP Alexandre SM, Nakamura MU. Assessment of pelvic floor of women runners by three-dimensional ultrasonography and surface electromyography. A pilot study.MedUltrason 2014; 16(1): 21-6.

20. Reis AO, Câmara CNS, Santos SG, Dias TS. Estudo comparativo da capacidade de contração do assoalho pélvico em atletas de voleibol e basquetebol. Rev Bras Med Esporte 2011; 17(2): 97-101.

21. Da Roza T, de Araujo MP, Viana R, Viana S, Jorge RN, Bo $K$ MascarenhaS T. Pelvic floor muscle training to improve urinary incontinence in young, nulliparous sport students: a pilot study. Int Urogynecol J 2012; 23:1069-73.

22. Rivalta M, Sighinol MC, Micali S, De Stefani S, Torcasio F, Bianchi G Urinary Incontinence and Sport: First and Preliminary Experience With a Combined Pelvic Floor Rehabilitation Program in Three Female Athletes. Health Care Women Int 2010; 31(5):435-43.

23. Carls $C$. The prevalence of stress urinary incontinence in high school and college-age female athletes in the midwest: implications for education and prevention. UrolNurs. 2007; 27(1):21-4

24. Roza TD, Brandão S, Oliveira D, Mascarenhas T, Parente M, Duarte JA, Jorge RN. Football practice and urinary incontinence: Relation between morphology, function and biomechanics. J Biomech 2015; 48(9):1587-92.

25. Luginbuehl $H$, Greter $C$, Gruenenfelder D, Baeyens JP, Kuhn A, Radlinger L. Intra-session test-retest reliability of pelvic floor muscle electromyography during running. Int Urogynecol J 2013; 24(9):1515-22.

26. Nygaard IE, Thompson FL, Svengalis SL, Albright JP. Urinary incontinence in elite nulliparous athletes. ObstetGynecol 1994; 84(2):183-7.

27. Eliasson K, Larsson T, Mattsson E. Prevalence of stress incontinence in elite trampolinists. Scand J Med Sci Sports 2002; 12(2):106-10.

28. Nygaard I, DeLancey JO, Arnsdorf L, Murphy E. Exercise and incontinence. ObstetGynecol 1990; 75(5):848-51.

29. Bo K, Borgen JS. Prevalence of stress and urge urinary incontinence in elite athletes and controls. Med Sci Sports Exerc 2001;33(11):1797-802.

30. Bo K, Sundgot-Borgen J. Are former female elite athletes more likely to experience urinary incontinence later in life than non-athletes? Scand J Med Sci Sports2010; 20:100-104.

31. CayletN, Fabbro-Peray P, Marès P, Dauzat M, Prat-Pradal D, Corcos J. Prevalence and occurrence of stress urinary incontinence in elite women athletes. Can J Urol2006; 13(4): 3174-9.

32. Fozzatti C, Riccetto C, Herrmann V, Brancalion MF, Raimondi M, Nascif $\mathrm{CH}$, Marques LR, Palma PP. Prevalence study of stress urinary incontinence in women who perform high-impact exercises. IntUrogynecol J 2012; 23(12): 1687-91.

33. Salvatore S, Serati M, Laterza R, Uccella S, Torella M, Bolis PF. The impact of urinary stress incontinence in young and middle-age women practising recreational sports activity: an epidemiological study. Br J Sports Med 2009; 43(14): 1115-8.

34. Kruger JA, Murphy BA, Heap SW. Alterations in levator ani morphology in elite nulliparous athletes: A pilot study. Aust N Z J ObstetGynaecol2005; 45(1): $42-7$.

35. Ree ML, Nygaard I, Bo K. Muscular fatigue in the pelvic floor muscles after strenuous physical activity. Acta ObstetGynecolScand 2007; 86(7):870-6.

36. Thyssen HH, Clevin L, Olesen S, Lose G. Urinary incontinence in elite female athletes and dancers. Int Urogynecol J Pelvic Floor Dysfunct 2002 13(1):15-7.

37. Merletti R, Parker PJ. Electromyography: physiology, engineering, and non-invasive applications. New Jersey: Wiley, 2004.

38. Júnior EA, Freitas RCM, Bella ZIKJ, Alexandre SM, Nakamura MU, Nardozza LMM, Moron AF. Assessment of pelvic floor by threedimensional-ultrasound in primiparous women according to delivery mode: initial experience from a single reference service in Brazil. Rev Bras GinecolObstet 2013; 35(3):117-122.

39. Dorschner W, Stolzenburg JU. A new theory of micturition and urinary continence based on histomorphological studies. 3 . The two parts of the musculus sphincter urethrae: physiological importance for continence in rest and stress. Urol Int 1994; 52(4), 185-8.

40. Kruger JA, Dietz HP, Murphy BA. Pelvic floor function in elite nulliparous athletes.Ultrasound ObstetGynecol 2007;30(1):81-5.

41. Dumoulin C, Hay-Smith J, Mac Habée-Séguin G. Pelvic floor muscle training versus no treatment, or inactive control treatments, for urinary incontinence in women. Cochrane Database Syst Rev 2014:14(5): CD005654

42. Bo K. Urinary incontinence, pelvic floor dysfunction, exercise and sport. Sports Med 2004; 34(7):451-64. 